GA-A22338

CONF-960543--44

\title{
A HYBRID DIGITAL-ANALOG LONG PULSE INTEGRATOR
}

\author{
by \\ E.J. STRAIT, J.D. BROESCH, R.T. SNIDER, and M.L. WALKER
}

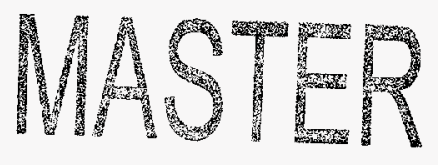

NISTRIEUTON OF TWS DOQUNENT IS UNLIMITED 


\section{DISCLAIMER}

Portions of this document may be illegible in electronic image products. Images are produced from the best available original document. 


\section{DISCLAIMER}

This report was prepared as an account of work sponsored by an agency of the United States Government. Neither the United States Government nor any agency thereof, nor any of their employees, makes any warranty, express or implied, or assumes any legal liability or responsibility for the accuracy, completeness, or usefuliness of any information, apparatus, product, or process disclosed, or represents that its use would not infringe privately owned rights. Reference herein to any specific commercial product, process, or service by trade name, trademark, manufacturer, or otherwise, does not necessarily constitute or imply its endorsement, recommendation, or favoring by the United States Government or any agency thereof. The views and opinions of authors expressed herein do not necessarily state or reflect those of the United States Government or any agency thereof. 
GA-A22338

\title{
A HYBRID DIGITAL-ANALOG LONG PULSE INTEGRATOR
}

\author{
by \\ E.J. STRAIT, J.D. BROESCH, R.T. SNIDER, and M.L. WALKER
}

This is a preprint of a paper to be presented at the 11th Topical Conference on High Temperature Plasma Diagnostics, May 12-16, 1996, Monterey, California and to be published in the Proceedings.

\author{
Work supported by \\ the U.S. Department of Energy \\ under Contract No. DE-AC03-89ER51114
}




\section{ABSTRACT}

A digital-analog integrator has been developed for use with inductive magnetic sensors in long-pulse tokamaks. Continuous compensation of input offsets is accomplished by alternating analog-to-digital convertor samples from the sensor and a dummy load, while an RC network provides passive integration between samples. Typically a sampling rate of $10 \mathrm{kHz}$ is used. In operational tests on the DIII-D tokamak, digital and analog integration of tokamak data show good agreement. The output drift error during a $1200 \mathrm{~s}$ integration interval corresponds to a few percent of the anticipated signal for poloidal field probes in International Thermonuclear Experimental Reactor (ITER), and bench tests suggest that the error can be reduced further. 


\section{INTRODUCTION}

Real-time discharge control and post-experiment data analysis in magnetic fusion devices require accurate measurements of the magnetic field just outside the plasma boundary. Inductive pickup loops 1,2 remain the primary choice for magnetic diagnostics in future tokamaks such as ITER, 3,4 because of their simplicity and durability. However, voltage signals from these loops need integration in order to obtain the value of the magnetic field. Active analog integrators are adequate for present pulse lengths of $10^{1}$ to $10^{2} \mathrm{~s}$, but anticipated pulse lengths of $10^{4}$ to $10^{5} \mathrm{~s}$ in future devices represent a challenge.

Analog integrators are limited primarily by variations in the input offset voltage which may occur during the pulse. The output voltage $V_{0}$ of an inductive loop is integrated to recover the magnetic flux which links the loop, $\Phi=\int V_{0} d t$. A spurious offset added to $V_{0}$ leads to a linear drift at the integrator output, which can become large when integrated over a long pulse. Magnetic fluxes $\Phi$ on the order of $0.3 \mathrm{Vs}$ must be measured in ITER, ${ }^{4}$ based on a typical poloidal field B of $1 \mathrm{~T}$ and a local magnetic field probe with a turns-area product (NA) of $0.3 \mathrm{~m}^{2}$. Accuracy of $2 \%(6 \mathrm{mVs})$ over a pulse length of $10^{4} \mathrm{~s}$ would require that the input offset of an analog integrator remain constant to within $0.6 \mu \mathrm{V}$ during that time, a stringent requirement.

Correction of an offset which varies with time requires that it be measured during the pulse. One proposed scheme ${ }^{5}$ alternates between two analog integrators, measuring the output drift in one while integrating the input signal with the other; a digital signal processor applies the measured drift correction and joins the two channels into a single output stream. We describe here a mostly digital integrator which provides essentially continuous correction of such offsets, and should be capable of accurate integration for very long pulse lengths. A prototype model $^{6}$ has been tested on the DIII-D tokamak. ${ }^{7}$ 


\section{METHOD}

The digital-analog integrator utilizes a passive $\mathrm{RC}$ integrator followed by an amplifier, analog-to-digital convertor (ADC) and real-time digital signal processor (DSP), as shown in Fig. 1. The RC time constant is long compared to the ADC sampling interval $\Delta t$, but short compared to the pulse length. The output voltage $V_{1}$ of the $R C$ network is given by $R C V_{1}=\int\left(V_{0}-V_{1}\right) d t$. Using samples $V_{i}$ of the $R C$ integrator's output voltage $V_{1}$, the real-time processor calculates the integral of the input voltage $V_{0}$, which equals the magnetic flux $\Phi$ through the pickup loop:

$$
\Phi=\int V_{0} d t=\int V_{1} d t+R C V_{1} \approx \Sigma V_{i} \Delta t+R C V_{i}
$$

where $\Delta t$ is the sampling interval of the ADC. Here $V_{i}$ is understood to represent the voltage at the amplifier input, so the amplifier gain $G$ does not appear explicitly in Eq. (1).

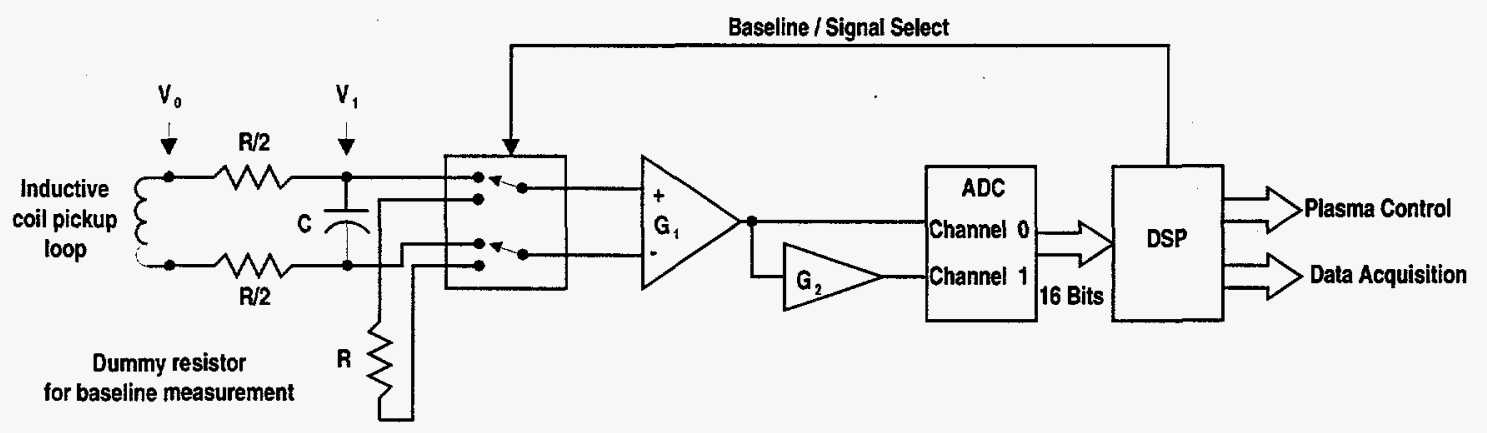

Fig. 1. Block diagram of digital-analog long-pulse integrator.

The passive $\mathrm{RC}$ integrator provides accurate integration of transients which are too rapid for the ADC sampling rate. For transients with a time scale shorter than the RC time, the RC integral [second term on the right-hand side of Eq. (1)] initially contains most of the integrated value. The digital integral (first term on the right-hand side) can be thought of as a correction for the slow decay of the passive integrator. On the other hand, for slowly varying signals the digital integral dominates and the $\mathrm{RC}$ term becomes negligible.

The gain $\mathrm{G}$ is chosen to match the signal to the dynamic range of the ADC. The flux may change by its full value $\Phi_{\max }$ on a time scale shorter than RC, for 
example in a disruption. The gain must be small enough to allow this without exceeding the voltage range $\mathrm{V}_{\max }$ of the ADC: $\Phi_{\max }(\mathrm{G} / \mathrm{RC})<\mathrm{V}_{\max }$. In practice, a second, high-gain channel is also included in order to improve the resolution of low signal amplitudes which occur during near steady conditions. The appropriate branch is chosen by the DSP for processing.

Continuous measurement of the time-dependent baseline offset voltage is obtained by switching the amplifier input between the output of the RC integrator and a dummy load with resistance $\mathrm{R}$ on alternate samples, as shown in Fig. 1. This correction is applied in real time, and the $V_{i}$ terms in Eq. (1) become $\left(V_{i}-U_{i}\right)$, where $V_{i}$ represent samples from the loop with $R C$ integrator, and $U_{i}$ represent baseline samples from the dummy load. The switch used for alternating signal and baseline samples may also introduce an offset, which cannot be measured by the same technique but must be measured before the beginning of the pulse and subtracted. In this case the $V_{i}$ terms in Eq. (1) become $\left[\left(V_{i}-\bar{V}\right)-\left(U_{i}-\bar{U}\right)\right]$, where $\bar{V}$ and $\bar{U}$ represent average values of $V_{i}$ and $U_{i}$ measured before the start of the tokamak pulse.

A small amount of noise superimposed on the signal is crucial to accurate integration, and must be added artificially if necessary. In the absence of noise, the finite voltage resolution of the ADC could lead to systematic errors. For example, large errors could occur when the magnetic flux to be measured changes steadily but at a rate so slow that the ADC input voltage is smaller than one count of the digital circuit, since the integrated output would remain unchanged. However, the addition of a few counts worth of random noise yields a measurable distribution of voltage samples with the appropriate fractional-count mean value, giving an effective sensitivity better than one count when averaged over many samples. Furthermore, the addition of noise allows the sample errors to be considered as random rather than systematic. 


\section{EXPERIMENTAL TESTS}

A prototype integrator has been built and tested during DIII-D operation. The signal from a passive filter with $\mathrm{RC}=0.01 \mathrm{~s}$ is fed to low and high gain amplifiers, with typical gains of 1 and 10. Data are acquired from both channels with a typical sampling rate of $10 \mathrm{kHz}$ ( $5 \mathrm{kHz}$ each for the signal and baseline samples), using a 16-bit $\mathrm{ADC}$ with a voltage resolution at $\mathrm{G}=1$ of $305 \mu \mathrm{V}$. The $5 \mathrm{kHz}$ switching rate requires the use of an analog switch rather than a relay for the alternation of signal and baseline samples. Signal processing is performed by a Texas Instruments DSP module, and an IBM-compatible PC is used for interfacing to the DSP module, application development, and data archival. This system allows both data processing in real time and post-acquisition analysis of archived data, for testing and comparing algorithms. The hardware configuration and some of the signal processing algorithms are discussed in more detail in Reference 6.

The problem of time-varying drift, and its alleviation by continuous baseline sampling, are illustrated in Fig. 2. In this bench test the high-gain channel $(\mathrm{G}=10)$ was used to integrate a zero input signal. The integrated signal without baseline correction [Fig. 2(b)] is initially zero, but after $400 \mathrm{~s}$ of integration begins to drift downward, apparently due to a change in offset voltage. This drift grows to an error of $10 \mathrm{mVs}$ by the end of the $1200 \mathrm{~s}$ integration time. This error could not be corrected in a conventional analog integrator, which would have an output similar to Fig. 2(b). However, here the digitally integrated baseline signal [Fig. 2(a)] tracks the offset change well, and the baseline-corrected integral [Fig. 2(c)] has an error of less than $1 \mathrm{mVs}$. The drift error becomes somewhat larger during DIII-D operational tests, typically reaching 5 to $10 \mathrm{mVs}$ during a $1200 \mathrm{~s}$ pulse. ${ }^{6}$ This is most likely a consequence of greater noise pickup in the tokamak operating environment (see Section III below). However, this error is still only a few percent of the anticipated signal for ITER, as would be required for an ITER pulse of similar length.

The accuracy of the digital integral during a tokamak pulse is shown in Fig. 3. The time history of the plasma current and neutral beam heating power are shown in Fig. 3(a), and the raw data for the integrator in Fig. 3(b). The raw signal includes a significant noise component, about $5 \mathrm{mV}$ during the tokamak pulse. 
Nevertheless a very accurate integral is obtained, as shown in Fig. 3(c), where the digital integral is compared with the output of an analog integrator connected to a similar poloidal field probe. Throughout most of the pulse, the two agree to within $1 \%$. A slightly greater disagreement near plasma initiation may be the result of slightly different poloidal locations for the two probes which are being compared.
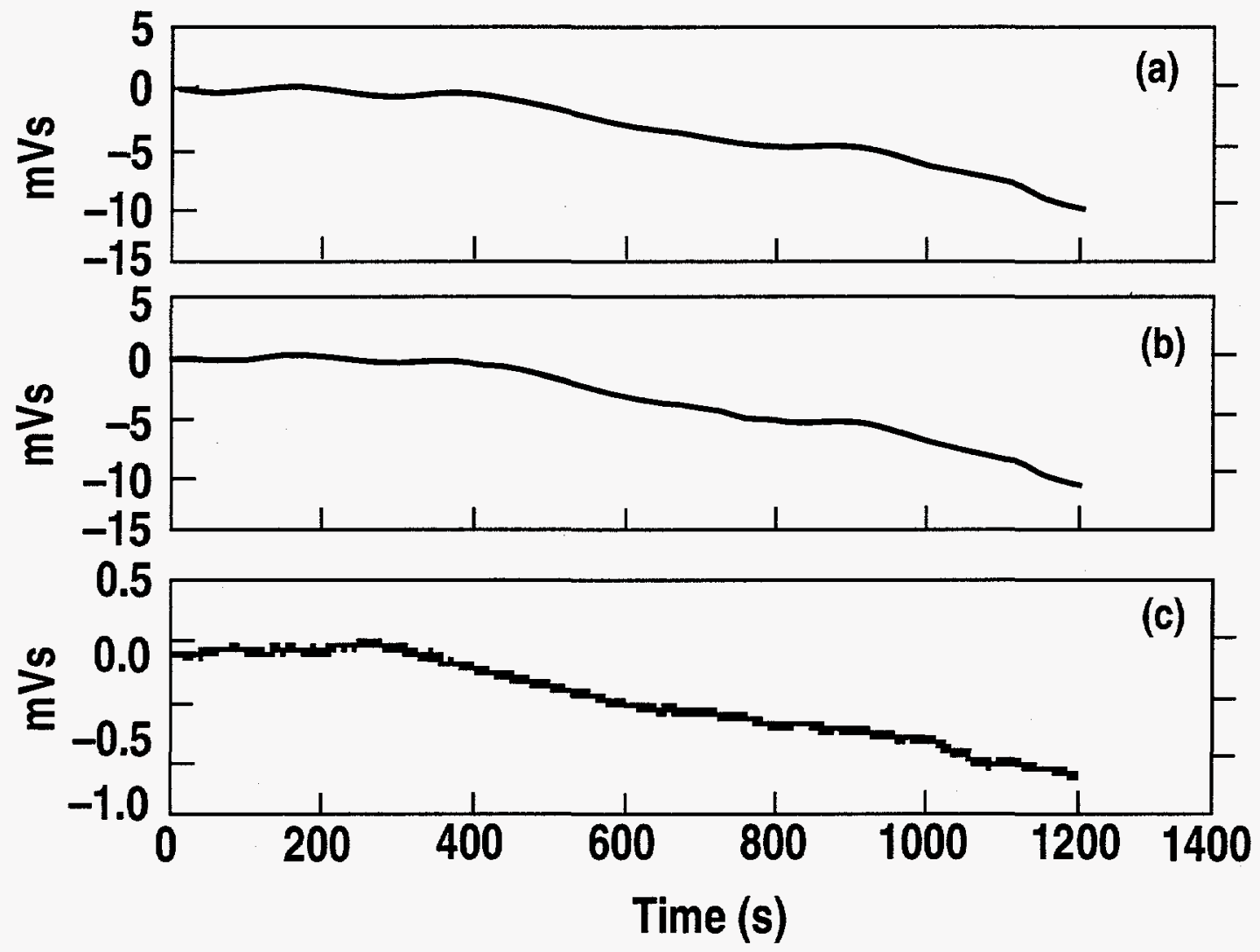

Fig. 2. Bench test of output drift reduction: (a) integrated baseline signal, (b) integrated input signal, (c) integrated input signal with baseline correction. 

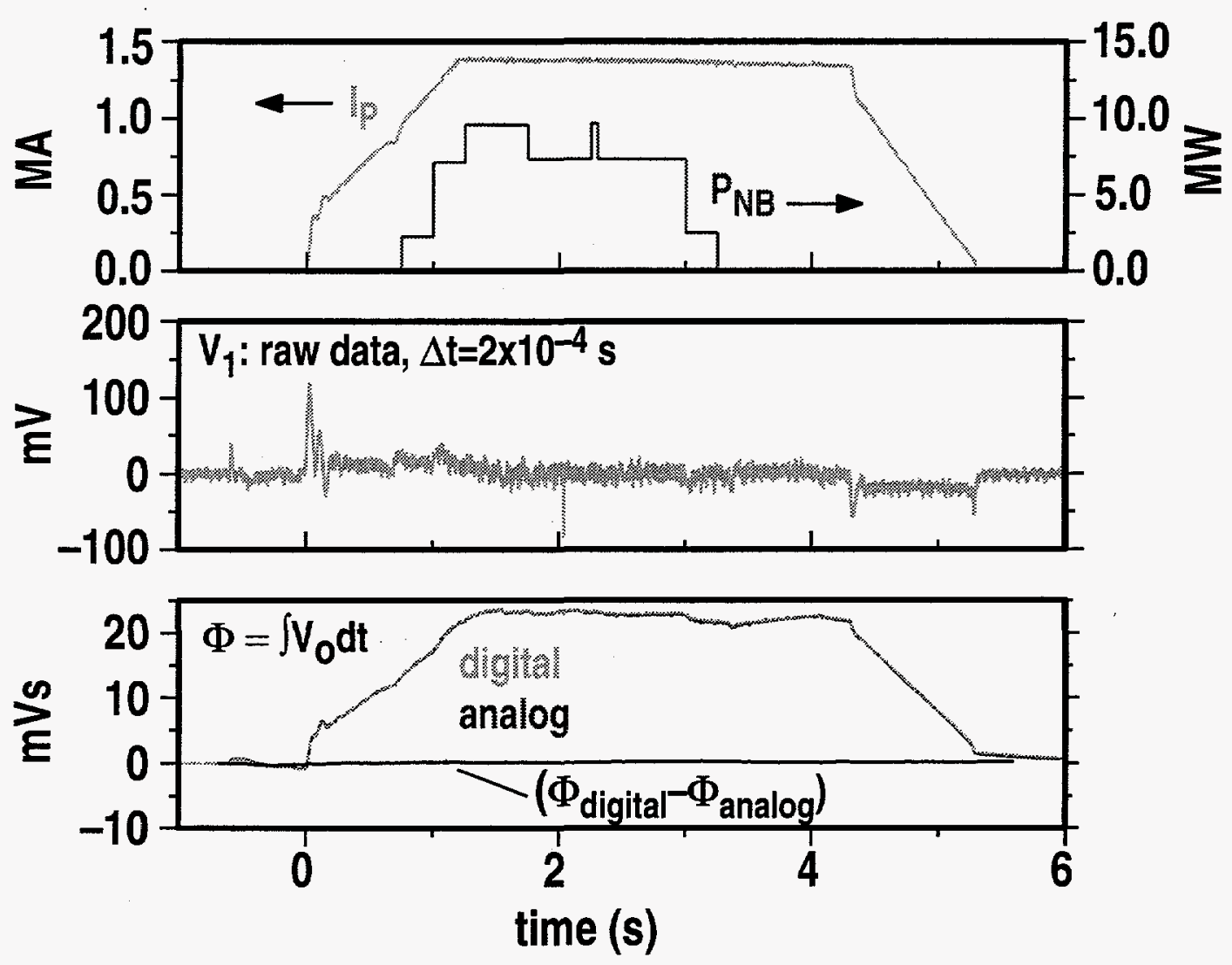

Fig. 3. Integration of a poloidal field probe signal during a DIII-D tokamak pulse: (a) plasma current and neutral beam power, (b) unintegrated probe signal, (c) digitally integrated probe signal (solid line), analog integrated signal from a similar probe (broken line, barely distinguishable), and difference of digital and analog integrals. 


\section{ERROR ANALYSIS}

The discrete nature of the digital integration introduces statistical uncertainties which are absent in an analog integrator. Furthermore, in an analog integrator, high frequency noise integrates to a low amplitude, but in a digital integrator the finite sampling rate aliases some of the noise spectrum to low frequencies where it has a larger impact on the integral. The uncertainty in the integrated value is dominated by the cumulative error in the summation term of Eq. (1), which in turn is assumed to be dominated by the noise component of the signal rather than the voltage resolution of the ADC. If the voltage samples are statistically independent with a standard deviation $\delta \mathrm{V}$ (due to noise, for example) then the uncertainty in the summation term is

$$
\delta \Phi=(2 \mathrm{~N})^{1 / 2} \delta \mathrm{V} \Delta \mathrm{t}=\delta \mathrm{V}(2 \mathrm{~T} \Delta \mathrm{t})^{1 / 2}
$$

where $\mathrm{N}$ is the total number of samples each of the signal and baseline, $\Delta \mathrm{t}$ is the sampling interval, and $\mathrm{T}=\mathrm{N} \Delta \mathrm{t}$ is the integration time. The factor of 2 results from summing uncertainties in the signal and baseline values. Even with continuous measurement of the baseline offset the uncertainty in the measured integral increases with time, but only as $\mathrm{T}^{1 / 2}$.

The experimentally observed integration error is consistent with Eq. (2). For the case shown in Fig. 3, the predicted error is $0.15 \mathrm{mVs}$ at the end of the tokamak pulse, which is consistent with the observed difference between the digital and analog integrals. The effect of noise, pulse length, and sampling rate is shown in Fig. 4. Here the integrator was operated with an unusually low sampling rate of $1 \mathrm{kHz}$, and a still lower rate was simulated in post-acquisition analysis. The noise level was also unusually large. Ideally, the integral is expected to remain at zero following the tokamak pulse seen early in the time history. The actual integral is compared with the uncertainty predicted by Eq. (2), using the measured rms noise amplitude $\delta \mathrm{V}=10 \mathrm{mV}$. It is clear that the error is larger for the lower sampling rate, increases with time, and in both cases remains within the expected uncertainty. 

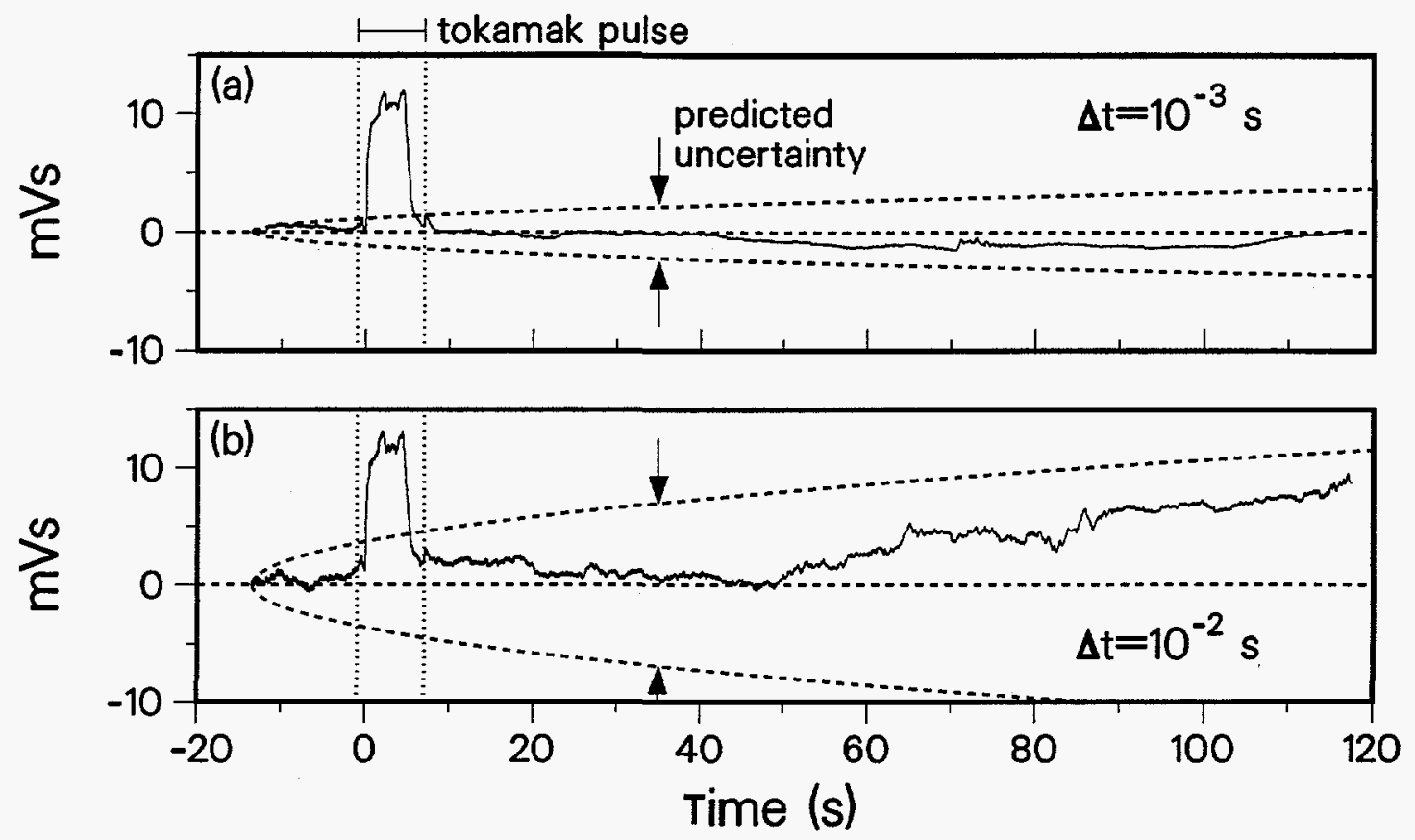

Fig. 4. Influence of noise on the accumulated error signal for different sampling intervals. The digitally integrated signal from the same data set is shown (solid lines) for sampling intervals of (a) $\Delta t=10^{-3} \mathrm{~s}$, and (b) $\Delta t=10^{-2} \mathrm{~s}$. The uncertainty $\delta \Phi$ (upper and lower broken lines) is estimated from Eq. (2) and plotted relative to the expected value of zero. The data set includes a tokamak pulse at $0<\mathrm{t}<5 \mathrm{~s}$.

Equation (2) imposes an upper limit on the pulse length, given that the noise amplitude $\delta \mathrm{V}$ must not be reduced to zero as discussed in Section II. The high gain channel with $\mathrm{G}=\mathrm{G}_{1} \mathrm{G}_{2}$ is used for most of the voltage samples, but the lower gain $\mathrm{G}_{1}$ determines the maximum flux $\Phi_{\max }=\mathrm{V}_{\max } \mathrm{RC} / \mathrm{G}_{1}$ which can be reliably integrated within the voltage range $\mathrm{V}_{\max }$ of the ADC, as discussed in Section I. If the tolerance for error is $\delta \Phi / \Phi_{\max } \leq \varepsilon$, then Eq. (2) leads to

$$
\mathrm{T} \leq\left(\varepsilon \mathrm{RC} \mathrm{G}_{2} \mathrm{~V}_{\max } / \delta \mathrm{V}_{\mathrm{c}}\right)^{2 / 2} \Delta \mathrm{t}
$$

where $\delta \mathrm{V}_{\mathrm{c}}$ is the noise amplitude at the ADC input, equivalent to $\delta \mathrm{V}=\delta \mathrm{V}_{\mathrm{c}} / \mathrm{G}_{1} \mathrm{G}_{2}$ at the high-gain amplifier input.

The pulse length can be maximized by reducing the noise amplitude $\delta \mathrm{V}_{\mathrm{c}}$ to a small (but non-zero) value and raising the gain $\mathrm{G}_{2}$, in order to increase the signalto-noise ratio. The pulse length can also be maximized by sampling at a faster rate or increasing $\mathrm{RC}$, but there are practical limitations to $\Delta \mathrm{t}$, and $\mathrm{RC}$ is constrained by 
the maximum flux $\Phi_{\max }$ which is to be measured. With the parameters of the DIII-D prototype and a minimum noise amplitude equal to one digitizer count $\left(\delta \mathrm{V}_{\mathrm{c} /} \mathrm{V}_{\max }=2^{-15}\right)$, a tolerance $\varepsilon=2 \%$ gives an absolute upper limit on the pulse length of $\mathrm{T} \leq 10^{7} \mathrm{~s}$. A more realistic amplitude of 10 digitizer counts, still only about $0.3 \mathrm{mV}$ at the high-gain amplifier input, yields $\mathrm{T} \leq 10^{5} \mathrm{~s}$, while typical noise amplitudes in the present DIII-D tests of $3 \mathrm{mV}$ yield $\mathrm{T} \leq 10^{3} \mathrm{~s}$. Because of the strong $\left(\delta \mathrm{V}_{\mathrm{c}}\right)^{-2}$ dependence, significant improvements in the performance of the digital integrator can clearly be realized from noise reduction, either through filtering or by reducing the ambient electromagnetic noise.

An additional uncertainty is introduced by subtraction of the initial measurement of the offset voltage $\bar{V}_{s}$ due to the switch. This offset is obtained by averaging over $N_{\mathrm{S}}$ samples before the start of the pulse: $\overline{\mathrm{V}}_{\mathrm{s}}=\overline{\mathrm{V}}-\overline{\mathrm{U}}=\Sigma\left(\mathrm{V}_{\mathrm{i}}-\mathrm{U}_{\mathrm{i}}\right) / \mathrm{N}_{\mathrm{s}}$. The uncertainty in this measurement is $\delta \overline{\mathrm{V}}_{\mathrm{s}}=\left(2 / \mathrm{N}_{\mathrm{s}}\right)^{1 / 2} \delta \mathrm{V}$. Because $\overline{\mathrm{V}}_{\mathrm{s}}$ is a fixed value to be subtracted from the integral, $\delta \bar{V}_{\mathrm{s}}$ represents a systematic rather than a random error, which leads to an uncertainty in the summation term of Eq. (1)

$$
\delta \Phi_{\mathrm{S}}=\mathrm{N} \delta \overline{\mathrm{V}}_{\mathrm{s}} \Delta \mathrm{t}=\delta \mathrm{V} \mathrm{T}\left(2 \Delta \mathrm{t} / \mathrm{T}_{\mathrm{S}}\right)^{1 / 2}
$$

where $T_{\mathrm{S}}=\mathrm{N}_{\mathrm{S}} \Delta \mathrm{t}$ is the time interval of this initial measurement. This uncertainty exceeds $\delta \Phi$ in Eq. (2) by a factor of $\left(\mathrm{T} / \mathrm{T}_{\mathrm{S}}\right)^{1 / 2}$ and furthermore increases with $\mathrm{T}$ rather than $\mathrm{T}^{1 / 2}$. If the noise amplitude $\delta \mathrm{V}$ is the same for both measurements then $\delta \Phi_{\mathrm{s}}$ represents the dominant uncertainty. However, if $\delta \mathrm{V}$ results from environmental noise associated with tokamak operation, then it may be possible to reduce $\delta \Phi_{\mathrm{s}}$ with a high-gain, low-noise measurement before the tokamak pulse. 


\section{DISCUSSION}

The digital-analog integrator described here is in principle capable of operation for very long pulse durations. Although the duration cannot be extended indefinitely, the integration error accumulates only as the square root of the pulse length.

Preliminary operational tests on the DIII-D tokamak have demonstrated the feasibility of the approach, with very good agreement between digital and analog integration of DIII-D tokamak data. Continuous baseline correction is shown to reduce the output signal drift by at least an order of magnitude compared to what would be seen in a conventional integrator having only an initial baseline correction. In bench tests, the drift error of $1 \mathrm{mVs}$ at the end of a $1200 \mathrm{~s}$ integration period was well within the required accuracy for an ITER pulse of similar duration, while in the noisier environment of tokamak operation the error was somewhat larger.

The noise component of the input signal is an important factor in the accuracy of the digital integral. A small amount of noise is needed to improve the voltage resolution for small signals, but as the noise increases further the accuracy becomes poorer. Experimental tests show that the accuracy of integration has the predicted dependence on the noise amplitude and sampling rate, supporting the expectation that control of the noise will lead to improvements in accuracy. 


\section{REFERENCES}

${ }^{1}$ R.H. Lovberg, in Plasma Diagnostic Techniques, eds. R.H. Huddlestone and S.L. Leonard (Academic Press, New York, 1965), Chapter 3.

${ }^{2}$ I.H. Hutchinson, Principles of Plasma Diagnostics (Cambridge University Press, Cambridge, 1990), Chapter 2.

${ }^{3}$ K. Tomabechi, J.R. Gilleland, Yu.A. Sokolov, R. Toschi, and the ITER Team, Nucl. Fusion 31, 1135 (1991).

${ }^{4}$ L. deKock, A. Costley, V. Mukhovatov, et al., "Overview of magnetic diagnostics planned for ITER," in Proceedings of the International Workshop on Diagnostics for ITER, Varenna, Aug. 28 - Sept. 1, 1995 (International School of Plasma Physics, Varenna), to be published.

${ }^{4}$ S. Ali-Arshad and L. deKock, Rev. Sci. Instrum. 64, 2679 (1995).

5J.D. Broesch, E.J. Strait, R.T. Snider, and M.L. Walker, "A digital long pulse integrator," General Atomics Report GA-A22218 (1995), Proc. 16th Symposium on Fusion Engineering, 1995 (to be published).

6J.L. Luxon and L.G. Davis, Fusion Technology 8, 441 (1985). 


\section{ACKNOWLEDGMENT}

This is a report of work supported by the U.S. Department of Energy under Contract No. DE-AC03-89ER51114. It was prepared as an account of work assigned to the U.S. Home Team under Task Agreement No. G 55 TD 09 within the Agreement among the European Atomic Energy Community, the Government of Japan, the Government of the Russian Federation, and the Government of the U.S. of America on Cooperation in the Engineering Design Activities for the International Thermonuclear Experimental Reactor (ITER EDA Agreement) under the auspices of the International Atomic Energy Agency (IAEA). 